\title{
Towards a cooperative approach for continuous innovation of Mobility Information Services
}

\author{
Dino Giuli, Senior Member, IEEE, Federica Paganelli, Member, IEEE, Stefano Cuomo, Paolo Cianchi
}

\begin{abstract}
Mobility Information Services (MIS), associated with Intelligent Transport Systems (ITS), are expected to provide valuable contributions towards sustainable and smart mobility. MIS inherit from ITS the characteristic of being a collaborative System of Systems (SoS) where no centralized management can actually be enforced and where heterogeneous types of independent actors are involved. Therefore, innovation in MIS cannot be conceived, represented and consequently promoted merely as a matter of advancements in specific technological fields. In this paper we propose a socio-technical framework for promoting continuous innovation in MIS. Focus is made on a cooperative approach, which leverages SoS principles, to foster service and product innovation in the mobility information domain at regional scale. We describe how this approach has been put into practice by architecting an "Open Working Environment". This is a socio-technical environment made by: $i$ ) a Cooperative Network of stakeholders (e.g., regional and national Public Administrations, Enterprises and Research Bodies); ii) an Architectural Framework, which is the reference technological baseline enabling the rapid configuration, deployment and integration of different technological capabilities. The latter is made also to support demonstration and validation activities of applied research results. Finally, we discuss obtained results, as well as future research directions and exploitation activities.
\end{abstract}

Index Terms - Intelligent transport systems; system of systems; mobility information services; service oriented architecture; socio-technical systems; cooperative systems; web services.

\section{INTRODUCTION}

$\mathrm{W}$ ITH the term "Mobility Information Services" (MIS) we refer to the broad range of services providing users with up-to-date and customized information and transactions for managing and supporting mobility of users themselves as well as transport of goods, potentially on a "door-to-door" scope and across heterogeneous transport modes.

Manuscript received March 28, 2012. This work was carried out within the SIMob project, funded by Tuscany Region.

D. Giuli is with the Electronics and Telecommunications Department, University of Florence, Florence, 50139, Italy (e-mail: dino.giuli@ unifi.it)

F. Paganelli is with the National Interuniversity Consortium for Telecommunications, Florence, Italy, 50139 (e-mail: federica.paganelli@ unifi.it)

S. Cuomo is with the National Interuniversity Consortium for Telecommunications, Florence, 50139, Italy (e-mail: stefano.cuomo@unifi.it)

P. Cianchi is with Negentis srl, Florence, 50110, Italy (e-mail: pcianchi@negentis.com)
As an example, MIS domains typically include: navigation, route planning, geo-referenced content delivery, alerts, payment and booking services, fleet management and goods monitoring services.

Mobility Information Services, associated with Intelligent Transport Systems (ITS), are expected to provide valuable contributions towards the attainment of sustainable mobility, by contributing to reduce the incidence of negative effects such as environmental pollution, accidents rates and congestions [1].

The European Commission [2] is promoting the development of Intelligent Transport System and Services to provide citizens with novel services, improve real-time and context-aware information delivery and management, while also helping public authorities and policy makers in rationalizing the use of available resources and in shaping proper policies for mobility.

The great number of research and experimentation projects in the Mobility Information Services domain proves that plenty of technological capabilities are purposely available in the sensing, communication, and information processing fields. Nonetheless, progress in the MIS domain is not only a matter of technological development. Several studies conducted in the last decades have analyzed the inherent complexity of ITS and MIS fields. Such a complexity is not only due to inherent functional and technological complexities, but also to organizational, socio-technical aspects and their interrelation at different geographical scales (e.g. local, regional, national scale). Moreover, the European Commission clearly states the need for supporting framework conditions to favor the introduction of new technologies without an appearance of picking winners [3].

Several studies [4],[5],[6],[7] have investigated how System of Systems (SoS) principles could help in modeling Intelligent Transport Systems and Mobility Information Services and analyzing determinants of innovation processes in these domains [8].

Moving from this theoretical background, our work aims at applying principles of SoS and socio-technical system design [9] to define and implement a framework fostering a cooperative approach towards innovation in MIS. This framework, named Open Working ENvironment for Mobility Information Services (OWEN-MIS), aims at facilitating the cooperation of stakeholders both through tangible (e.g. products and systems integration) and intangible (e.g. 
knowledge exchange) channels. Main constituents of the OWEN-MIS are: i) a Cooperative Network of stakeholders, including Public Administrations, Enterprises and Research Bodies; and ii) an Architectural Framework that provides the reference technological baseline enabling the rapid configuration and deployment of technological capabilities.

In particular, we developed such an approach to allow: i) a proficient support for applied research, also for demonstration and validation purposes; ii) fast and customized technology transfer into operational MIS.

Purposely, an experimental implementation of the OWENMIS has been carried out on a regional scale by applying the architectural framework guidelines for the deployment of an "Evolutionary Test-bed". We focused on a regional scale for the following reasons: i) these activities have been carried out within the SIMob research project, funded by Tuscany Region in the framework of a long-term initiative towards sustainable mobility in Tuscany; ii) the regional scale is to be considered significant in stimulating MIS innovation processes on a SoS perspective.

The paper is structured as follows: Section II offers a background on studies on SoS models and their application to ITS and MIS domains and motivates our work; in Section III we introduce the Open Working Environment and describe its main constituents. In Section IV we report on the implementation of the OWEN-MIS within the SIMob project. Concluding remarks are made in Section V.

\section{SYSTEM OF SYSTEMS IN THE ITS AND MIS DOMAINS}

According to the widely-accepted definition provided by Maier [5], a System of Systems (SoS) is an assemblage of components that can be individually regarded as systems and are characterized by operational and managerial independence and evolutionary behavior. The combination of such independent system components into a SoS performs a function that is not performable by a single component [10]. Evolutionary development and emergent behavior are considered as properties unique to SoS deriving from the managerial and technical autonomy of individual constituents.

Due to their inherent characteristics, SoS can be characterized as follows [11]:

- Components of a SoS are operationally autonomous; this implies that they have to pursue their own goal, independently and in addition to contributing to the global SoS objective(s).

- These components are often conceived, developed and managed independently.

- The behavior of a SoS cannot be fully predicted from the behavior of its component system.

Maier mentioned ITS as a relevant example of a SoS. In [6] he states that "In the ITS example the deployed collective of systems represents a mixed equilibria of social and technical factors. Private consumers and public agencies can only buy and deploy the elements technically available, but technical feasibility, even technical construction, is no guarantee the elements will be used. Moreover, the ways in which those elements will be used is unknown to the designers".

Consequently, while it is widely recognized that technical interfacing is a key aspect in determining SoS functioning, it has to be stressed that the operation of ITS and, as a consequence, of MIS, strongly depends also on social and economic aspects, such as private choice and public policies.

Ottens et al. [7] analyze ITS as examples of complex infrastructure systems strongly characterized by the intertwining of technical and social aspects. As social elements such as laws, regulations, policies, economic and organizational structures may influence the functioning of the system, Ottens et al. [7] discuss about the need of explicitly identifying and analyzing these elements when designing socio-technical systems.

Tuominen and Ahlqvist [12] focus on the challenges in designing transport policies that effectively take into account the rapid evolution in information and communication technologies. To this purpose, they propose a socio-technical roadmapping method and discuss the results obtained by testing the method within a Finnish case study.

A specific contribution on the study of innovation processes in ITS is provided by Mostafavi et al. [8]. In this work, the authors propose a novel an analysis framework for System of Innovation (SoI) studies, called I-SoS Framework. System of Innovation studies aim at understanding innovation processes by adopting system analysis principles. With respect to previous conceptualizations that model a SoI as a monolithic system [13], Mostafavi et al. [8] characterize a SoI as a SoS, i.e., "as a set of individual monolithic systems that interact to obtain functionalities that cannot reside in the individual systems". Innovation policy making in the area of ITS is used as an example for illustrating the application of their framework, since "the process of development and diffusion of ITS innovation cannot be performed by individual organization(s) and is the emergent result of activities and interactions among different organizations". Instead, ITS innovation should be properly modeled by representing interrelationships and dynamic boundaries across different Systems of Innovation, typically distinguished in Regional, National and Sectoral SoIs.

\section{A. Cooperation and information sharing as MIS enabling factors}

The provision of Mobility Information Services has to account for basic reference requirements coming from both front-end and back-end users. It has thus to consider mobility as a matter of transportation as well as a relevant aspect of daily living over the territory. Such personal mobility dues and needs call for information services which can utmost support people in both the pre-trip and on-trip phases.

Different actors typically provide specific mobility information services within their own systems. Their networking and cooperation is needed to enhance MIS provision as needed to cope with the above mentioned user requirements.

A basic need for the expected MIS evolution is information 
sharing among such actors. Open Data approaches are actually pushed for such a purpose.

A reference scenario of MIS concerned with all these aspects is sketched below.

\section{1) Transportation and territorial living personal needs} for MIS provision

Evolution of MIS has to account for some factors basically influencing specific real needs of users. These factors are significantly related with the following aspects:

- mobility and related information services are to be primarily oriented according to the people needs, as connected with the local area where they are living or going to live (even temporarily);

- most of such local areas are urban areas, where mobility is often a complex task;

- such complexity is enhanced by relevant connections with mobility at regional scale, including long-range mobility.

A mobility view which can be associated with the abovementioned scenario is reported in Fig. 1. It points out different types of mobility corresponding to different goals, which influence the characteristics of MIS to be correspondingly provided to the users. Main specific types of mobility are reported in Fig. 1, grouped within three macro-categories: basic daily mobility; short occasional stays of incoming people; mobility of local residents. As an example, we also highlight how these mobility types typically pertain to different geographical scales. We purposely distinguish three main levels of geographical coverage [14]: i) local coverage, meaning transport internal to urban areas or within an area surrounding a built-up area; ii) regional coverage involving the movement of passengers and freight between neighboring urban agglomerations and between them and their hinterlands; iii) long distance implying mobility over a considerable distance, across regional and national borders.

Therein we also roughly highlight how mobility goals and personal needs are expected to be supported by a variable

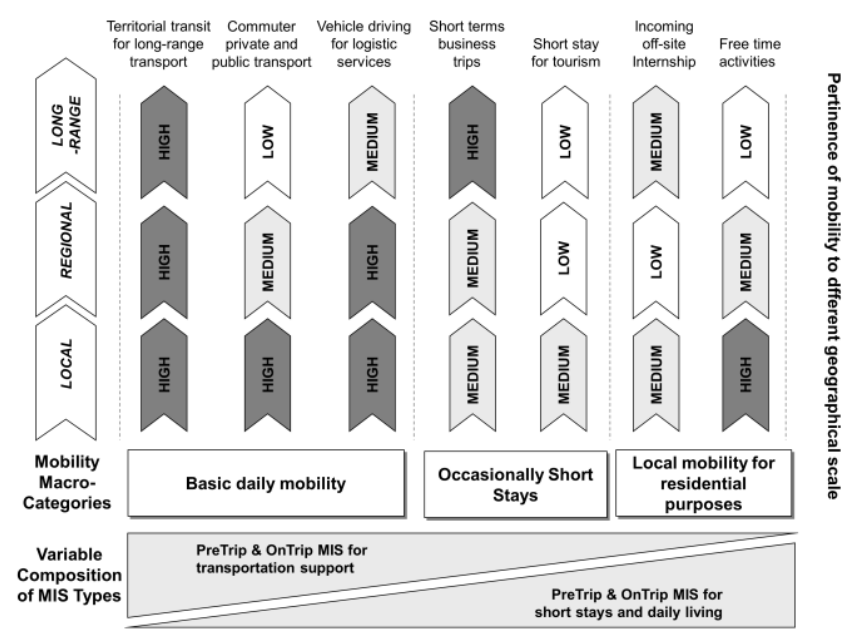

Fig. 1. Mobility macro-categories and related needs for Mobility Information Services provision composition of two different macro-types of MIS: i) pre-trip and on-trip MIS for transportation (e.g., route planning, up-todate information on planned itineraries, etc.); ii) pre-trip and on-trip MIS for occasional short stays and daily living (e.g., location-based, context-aware and personalized services on Point of Interests for tourism and free-time activities).

2) Towards information sharing and cooperative networking among MIS actors

It is widely recognized that models for cooperation and information sharing across actors involved in the Mobility Information domain are needed in order to provide reliable, exhaustive and cost-effective information services capable of supporting such complex and heterogeneous goals.

Different types of actors in the Mobility Information sector manage their own information systems for attaining their business/institutional goals. These systems are typically complex and characterized by a long-term life-cycle. Additional complexity has also to be managed for information systems bounding to a "physical infrastructure" (e.g. road infrastructure, public transport networks, etc.). This situation may lead to many inefficiencies, e.g., similar information may be collected by different bodies, while information that could be jointly and advantageously exploited by several actors is not actually shared. In some cases, actors may even be unaware that potentially useful information is being collected by other actors. Instead, cooperative networking is needed to promote and ease the conception and provision of addedvalued MIS.

In Fig. 2 we identify a non-exhaustive list of typologies of actors managing information systems that are expected to be networked and evolving for cooperation purposes, through information sharing and service integration. From the bottom to the top of the figure, we highlight some characterizing aspects of identified actors. At the bottom, we show actors that, to achieve their organizational goals, handle information systems controlling and monitoring infrastructural assets (including transport infrastructures, transport means, vehicular sensing and controlling hardware). Upwards in the figure, we list types of actors that handle information at progressively higher levels of abstraction, i.e. processing raw data and converting them into meaningful and valuable information assets. Identified categories span from transport infrastructure operators and operators of intermodal terminals and transport access nodes (e.g. ports, railway stations, bus stops) to providers of environmental monitoring and forecasting services, traffic and mobility operations centers, commercial content providers and providers of valued-added integrated services.

This perspective is strengthened by two enabling factors: i) the standardization efforts towards architectural guidelines and information exchange specifications (e.g., SIRI [15] and Datex2 [16]); ii) the increasing interest towards the adoption of "Open Data" approach in MIS as well as in other application domains. 


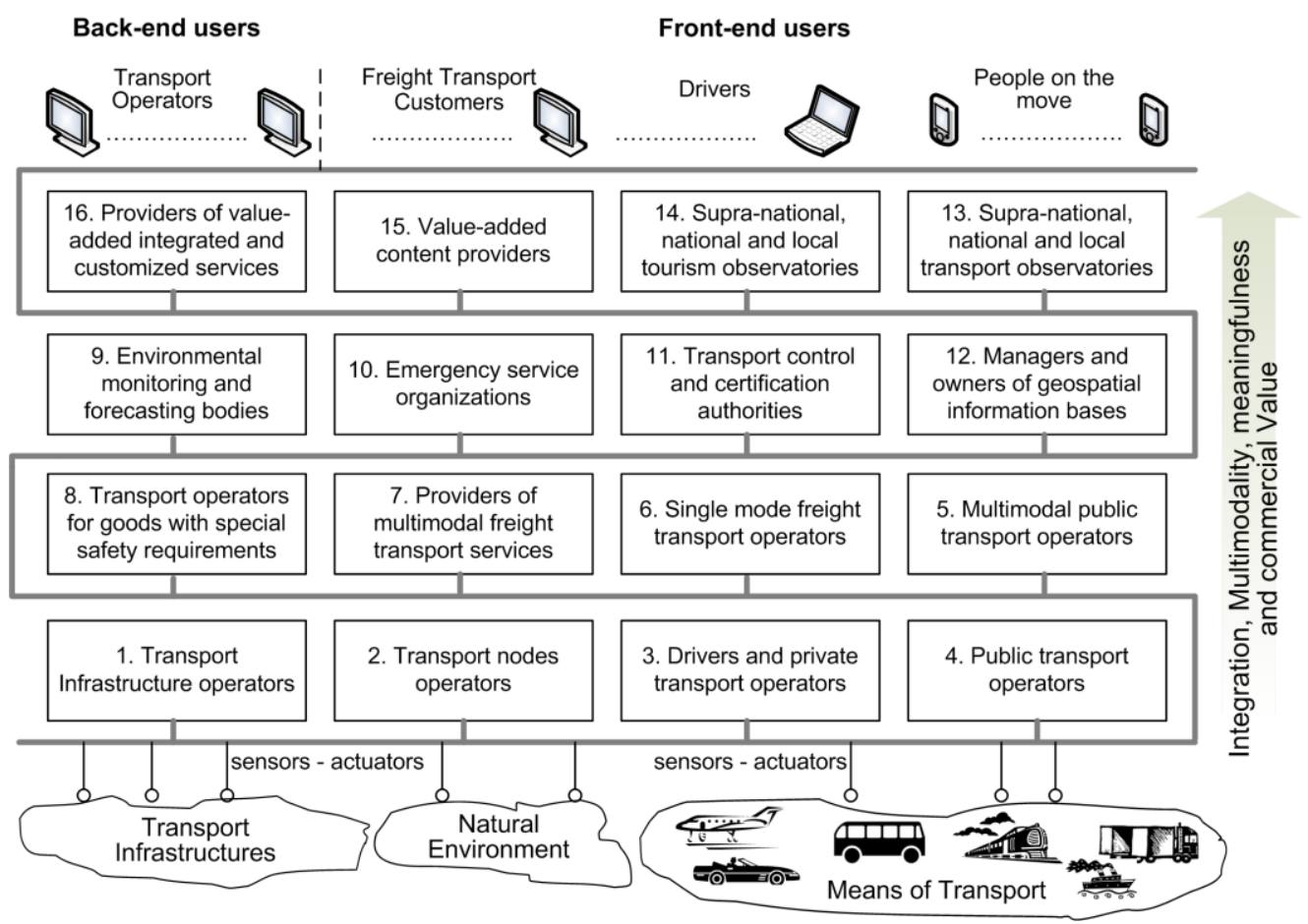

Fig. 2. Main actors in the Mobility Information Service domain

\section{3) Architectural guidelines}

The need for integrated Intelligent Transport Systems has motivated the work of several national and international work committees. A widely recognized approach is the development of a framework architecture, i.e. a set of architectural guidelines and interface specifications, from which specific ITS architecture implementations could be derived [5].

Relevant examples of national ITS architecture specifications are the ones produced in the USA [17], in Japan [18] and in China [19]. Analogously, the European Commission has released the European ITS Framework Architecture (the FRAME Architecture) [20], from which national ITS architecture specifications have been derived in several European countries (e.g., ARTIST in Italy [21], ACTIF in France [22], and ARKTRANS in Norway [23]).

These framework architectures share a common approach in providing guidelines and top-level specifications of a system structure. Several works have derived from these architectural guidelines hands-on approaches for the development of integrated ITS systems. For example, Coopers [24], CVIS [25] and In-Time [26] are European projects that have adopted and enhanced such guidelines for the implementation of technological advancements and novel services in the ITS domain.

\section{4) The "Open Data" approach}

"Open Data" refers to models for data publication and reuse promoting open information exchange and reuse across data producers and consumers. While the notion of "Open Data" is quite recent and not yet mature, a satisfactory definition is provided in [27]: "A piece of content or data is open if anyone is free to use, reuse, and redistribute it - subject only, at most, to the requirement to attribute and share-alike."

Open Data sharing initiatives are currently being promoted in several application domains (e.g., science, bibliographic data, and government). Indeed, several research bodies, national governments and local administrations, are publishing data sets on the web (e.g. data on education, health, safety, and transport, just to mention a few).

In this perspective, the European Commission has recently published an agenda for an "Open Data Strategy" suggesting key measures for promoting the widespread adoption of open data principles (i.e., legislative frameworks, creation of European data portals, financing instruments) [28]. The adoption of open data principles is thus expected to have a great impact in the ITS domain. Indeed, the availability of an increasing amount of geospatial data, travel and real-time traffic information is expected to be a key enabler for entrepreneurial innovation initiatives, by easing the conception and delivery of novel value-added MIS.

\section{B. Research Questions and Main Contribution}

The domain of MIS inherits from the one of ITS the characteristic of being a collaborative SoS, i.e., a SoS where no centralized management can actually be enforced and the SoS emerges from the fact that constituent elements collaborate, more or less, voluntarily.

As a matter of fact, actors of the MIS domain (e.g. public institutions and private organizations as shown in Fig. 2) can operate independently and actually operate autonomously, of course within the framework of regulations and standard specifications.

The MIS, considered as a SoS, and its autonomous 
components can be characterized as socio-technical systems, i.e., systems where not only technical elements (e.g. software and hardware technologies) but also people and their interrelations are involved to different extents and under different roles.

Moving from this theoretical background, our work aims to put into practice principles of SoS and socio-technical system design and analysis in order to foster service and product innovation in the area of Mobility Information Services. The work has been driven by the following research questions:

- Which actors are involved in innovation processes in the MIS domain?

- Why do these "autonomous" actors decide to collaborate and how reasons for collaboration can be fostered?

- How can networking and cooperation among autonomous actors and related systems be effectively supported and exploited in order to promote MIS innovation?

\section{OPEN WORKING ENVIRONMENT}

The main contribution of this work consists in the definition and experimentation of a cooperative approach to enable continuous innovation in the Mobility Information Service domain. We labeled this approach "Open Working Environment for Mobility Information Services" (OWENMIS).

With this term we refer to an organizational, scientific and technological environment, whose objective is to facilitate the cooperation of stakeholders towards an effective continuous innovation in the MIS domain. We can characterize the OWEN-MIS with the steady and mutual interaction of two main entities:

i) a Cooperative Network of stakeholders who are jointly involved, to a different extent, in the conception, research, development, exploitation and governance of mobility information solutions. This network is thus open to regional and national subjects from Public Administrations, Enterprises and Research Bodies.

ii) an Architectural Framework, providing the reference technological baseline for effectively promoting innovation by enabling the rapid configuration and deployment of MIS. The objective of this framework is therefore to provide methodologies, tools, reference implementation and crosssectorial technological functionalities to ease research, development and testing of novel solutions. This framework should thus enable the configuration of different technological environments fulfilling specific application purposes (e.g., application pilots, integration tests).

Fig. 3 shows the main interactions driving the behavior of the OWEN-MIS environment at a macro-level of analysis:

- From the Cooperative Network to the Architectural

Framework. The Cooperative Network acts on the Architectural Framework for the following main purposes: carrying out research and technology development activities; promoting the adoption of the framework as a reference environment for standard specifications and

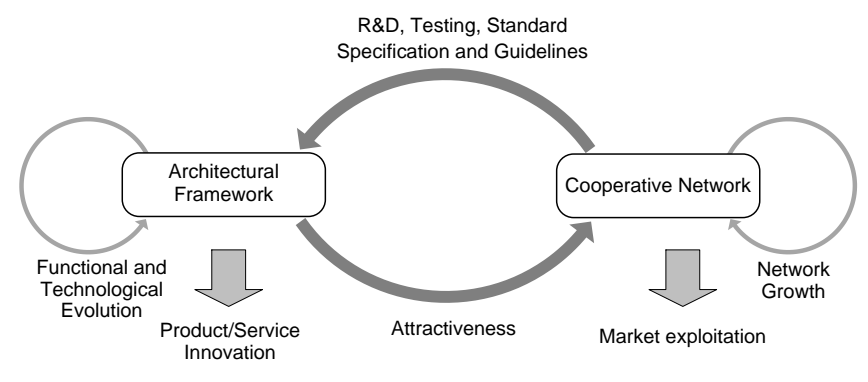

Fig. 3 Open Working Environment for Mobility Information Services (OWEN-MIS)

guidelines compliance and local needs and requirements fulfilling. This interaction can thus foster the functional and technological evolution of the Architectural Framework.

- From the Architectural Framework to the Cooperative Network. As long as the Architectural Framework evolves by offering novel functional and technological capabilities, it becomes progressively more attractive to Cooperative Network and external stakeholders, thus favoring the growth of the Network itself.

From the sketched interactions it emerges that the Architectural Framework aims at offering a set of configurable and extensible technological assets, helping the stakeholders in speeding up the design and development of novel sub-systems and systems, while promoting reuse, adaptation and integration with legacy systems and compliance with standard interfaces.

The Cooperative Network is thus called to support the development of the Framework, to exploit the technological layer for researching and developing novel prototypes and carrying out market exploitation activities, as well as to implement policies for promoting and strengthening the adoption of the framework itself.

Mutual interaction among the OWEN-MIS elements is expected to lead both to a growth of the capabilities offered by the framework making it more valuable, and to increase the attractiveness for new stakeholders to join the network, thus enhancing the overall skills and expertise of the network.

The main outcomes of this process can be regarded as product/service innovation, as fostered by the Architectural Framework's capabilities, and its exploitation from the Cooperative Network.

\section{A. Cooperative Network}

The Cooperative Network is participated by actors that are involved with different roles and expertise in the Mobility Information Service domain.

Main targeted actors are from Public Administrations (e.g. Municipalities, Regional and National Administrations), Enterprises (e.g., SMEs and big firms, ICT and Transport operators) and Research Bodies (Universities, Research Centers).

Table I details the role played by the Cooperative Network actors in the OWEN-MIS, while Fig. 4 highlights the interactions among different actor types, and how such interactions can be favored by the capabilities provided by the 


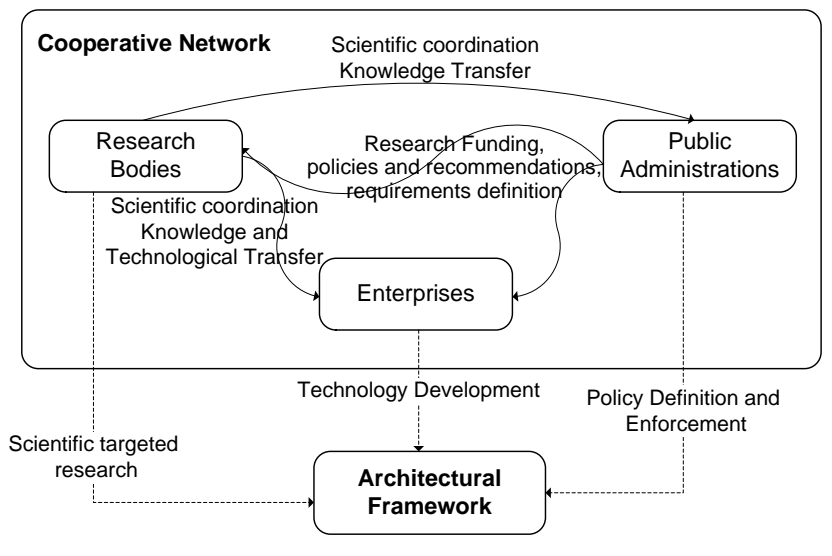

Fig. 4 The Cooperative Network

Architectural Framework, acting as a "technological enabler".

Public Administrations can promote policies for production and adoption of standards in the MIS domain (especially specifications for interoperability and message exchange). Thus, the Architectural Framework can be properly shaped in order to adhere to such standards and act as a reference implementation for these specifications.

Public Administrations can also play an active role as providers of open data (e.g., public transport information, realtime traffic data, road status information), thus contributing to favor third parties' innovation in the MIS domain. They can further contribute by supporting and funding research and networking initiatives and explicitly recognizing their role of excellence in research, development and technology transfer.

Enterprises are actors primarily involved in innovation processes. The combined efforts of firms with complementary expertise and business interests can favor and speed up the conception and realization of novel product and services [29] This approach can be especially advantageous for small and medium enterprises (SMEs) by promoting their organic networking. This latter facet is extremely important in Europe [30], and particularly in the Tuscany region (the area of interest for the SIMob project), since its economic landscape is majorly populated by small-sized enterprises in almost any domain, including the ICT and MIS [31]. In the envisaged cooperative innovation process, enterprises can leverage on the capabilities provided by the Architectural Framework for speeding up research, integration and testing activities.

Finally, Research Bodies contribute with their specific know-how and excellence in research activities. Collaboration with enterprises can be achieved via technological transfer initiatives and start-up of joint laboratories and research spinoffs. Research Bodies can leverage on the Architectural Framework as a basic infrastructure for carrying out mediumand long-term research. Moreover, they play a relevant role in the scientific coordination of the Network by identifying and specifying scientific and technological guidelines and in assessing quality of the overall results.

Based on the considerations mentioned above, in Table I we also highlight main expected outcomes that can foster reasons for cooperation in the Network.

\section{B. Architectural Framework}

The Architectural Framework is a technological framework devised as a common working infrastructure for the Cooperative Network.

Its objective consists in supporting the development of novel ICT solutions for mobility, by easing the verification and testing of their interoperation with external systems since from the conception phase. Therefore, it is not aimed at providing

TABLE I

STAKEHOLDERS ROLE AND EXPECTED OUTCOMES IN THE COOPERATIVE NETWORK

STAKEHOLDERS

ROLE

EXPECTED OUTCOMES

- Contribution to the establishment and evolution of the
Architectural Framework
- Participation to research and product innovation processes

Enterprises
- Increased market opportunities also through the promotion of public and private demand of value-added services

- More favorable conditions fostering the establishment and competitiveness of SME networking for infomobility

- Increased quality and competitiveness of own industrial products

- Contribution to the establishment and evolution of policies for transport and ICT applications for transport

\section{Regional and}

Local Public Administrations

- Active role in defining requirements and transferability of results in regional/local policies and actions

- Provisioning of Open Data services

- Promotion of funding programs for research and development projects and knowledge and technological transfer actions

- Support actions for broadening and strengthening the Cooperative Network

- Scientific co-ordination of the Cooperative Network

- Research contribution for mobility product and service innovation in industrial and public administration context

Research Bodies
- Diffusion and evolution of value-added MIS at regional/local level

- Possible availability of a reference technological framework for recommended and standard guidelines

- Increase of business opportunities for local enterprises (especially SMEs and SME networks)

- Increased quality and transferability for scientific research results in the mobility information service field - New perspectives in academic education and scientific research through the technological environment made available by the Architectural Framework Start-up of public/private joint laboratories to support the development of the Architectural Framework with reference to specific technological sub-systems 
services for hosting system components and/or enabling information exchange during their operational lifecycle.

It should provide flexible and configurable services for supporting targeted research, design and development activities, as well agile experimentation of results by each actor side. More precisely, this working infrastructure has to fulfill the following main high-level requirements:

a) facilitating and speeding up the development of novel functional components by making common services and infrastructures available;

b) providing a reference framework enabling a preliminary validation of the interworking and integration of heterogeneous functional components;

c) providing support for the rapid design and development of pilot applications;

d) easing the adoption of horizontal and/or vertical standard specifications, with special focus on specifications whose adoption is recommended by authorities at local, regional and/or national level.

While focusing on a pre-operational phase and not on the deployment and operational phases of components in real-life scenarios on a SoS perspective, the Architectural Framework should mediate the interoperation across system components that are characterized by technological and managerial autonomy.

According to the SoS principles, the need of interoperation among heterogeneous and autonomous components is of key relevance, as it is the factor driving the evolutionary development and the emergent behavior of a SoS. Interfaces are first-class entities in a SoS, as they enable information exchange across SoS constituents.

Service Oriented Architecture (SOA) is widely considered as a paradigm that can fit well with $\mathrm{SoS}$ requirements [11], [32]. SOA is a paradigm for conceiving distributed applications by leveraging on the loosely coupled interactions among services. Functional and non-functional properties of service interfaces are described in standardized contracts. At present, SOA is considered "an architectural approach that is consistent with many of the needs of systems of systems. It involves methods and approaches that may be more generally applicable to systems of systems" [32].

Many current instantiations of SOAs are based on Web Service specifications, and common practices in WS-based development typically support static peer-to-peer interaction among service client and providers. Therefore, WS* specifications should be complemented with a manageable and flexible infrastructure [33].

We thus decided to build the target framework by leveraging on the highly distributable and extensible integration and message exchange capabilities provided by an Enterprise Service Bus (ESB) [34]. ESBs are open standardbased message-based backbones providing communication, message routing, resource discovery and integration capabilities. ESBs ease the implementation of distributed applications based on service-oriented and/or event-driven models, as they specify a service-oriented middleware with a message-oriented abstraction layer atop different messaging protocols (e.g., SOAP, JMS, SMTP). Rather than providing a direct one-to-one mapping between two messaging protocols, a service bus is an intermediary messaging backbone offering an $\mathrm{N}-1-\mathrm{M}$ mapping.

Data sources and application components from different providers can thus be modeled as operational nodes whose capabilities are exposed as service interfaces implementing message-exchange patterns, defined in well-specified contracts.

More specifically, an ESB can typically support different levels of integration:

- Data-level integration: it can provide a uniform access to heterogeneous data sources. Typically, special components, named adapters, are used to encapsulate legacy interfaces into standard and/or agreed message-based interfaces [35]. The adapter logic decouples the message-based interfaces from the specific interfaces and implementations offered by external components. This approach favors the extension and evolution of the platform by easing the addition of novel components as well as the extension and reconfiguration of the existing ones;

- Application-level integration: it provides the capability of plugging and making available functional capabilities provided by different software components;

- Process-level integration: it enables the specification of complex collaborative solutions as an automated process that integrates heterogeneous software component capabilities and human tasks. By relying on service orchestration capabilities, the system eases the specification of highly configurable processes that orchestrate and manage human tasks and services.

While ESBs provide flexible instruments to achieve interoperability with different levels of coupling among components and thus represent the state of the art in SOA implementations, still their evolution is required in order to effectively support interoperation across complex systems [36]. As discussed by Thomas et al. [36], interoperation challenges arise from the need of handling the extreme level heterogeneity as well as the increasing level of dynamism encountered in the systems called to interoperate.

Such complex requirements of a SoS are due to its inherent characteristics, as briefly discussed in Section II. It has been recognized that a viable approach to tackle some of these challenges would consist in enhancing SOA principles and specifications with autonomic models and with formal analysis and verification techniques [11].

To favor the inner evolution of the Architectural Framework, while avoiding technological lock-ins in the message-exchange platform, we decided not to leverage on a single ESB product. We thus adopted two ESB implementations:

- a COTS ESB made available by an industrial partner of the SIMob project (http://www.negentis.com). This ESB solution offers up-to-date design-time orchestration and 
composition capabilities, business process description, execution and monitoring capabilities. Further advantages are the compliance with widely accepted industrial standards (e.g., JMS [37], BPMN [38] and Web Services [39]) and availability of stable releases.

- a service-oriented middleware embedding results of recent and ongoing research activities through an incremental cycle of development and delivery of snapshot releases. In this context, research objectives consist in achieving: 1) an extreme degree of configurability; 2) dynamic service discovery, invocation and composition capabilities [40]; 3) specification and enactment of distributed security policies. We refer the reader to [41] for a detailed description of recent results in the design and development of this middleware.

Finally, the adoption of open and standard interfaces is considered as a strict requirement for adding novel functional components into the Architectural Framework. This is done to ease the development of adapter components for invoking provided functionalities as well as their different implementations and configurations over time, possibly provided by different actors.

Fig. 5 depicts how the Architectural Framework has been implemented, as described in the following section.

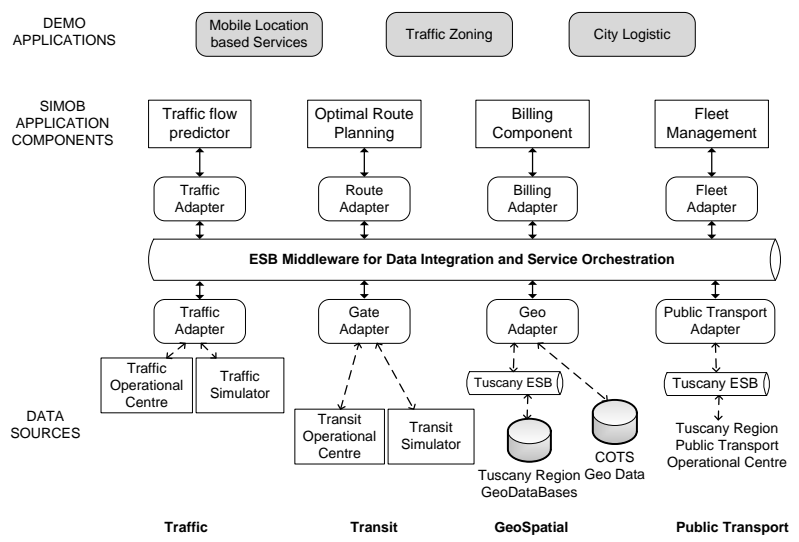

Fig. 5 Evolutionary Test-bed

\section{EXPERIENCES IN THE SIMOB PROJECT}

The core implementation of this approach has been carried out in the Tuscany Region, Italy, in the framework of SIMob (Sistema Integrato per l'infoMOBilità), a three-year research project (2008-2011) co-funded by the Tuscany Regional Administration [20]. The project activities were coordinated by the National Inter-University Consortium for Telecommunications (CNIT) and participated by representatives from main actors of ITS and MIS domains in Tuscany, including Universities and Research Centers (three university departments and an interuniversity consortium), SMEs with specific expertise (middleware, geo-spatial databases, logistic applications) and big firms (including two ICT industries and one highway operator). Public
Administrations were represented by Tuscany Region Government.

The project partners have thus agreed in establishing a Cooperative Network at regional level. Short-term objectives of the Network consisted in the deployment of a first instance of the Architectural Framework and the development of prototypal applications towards market exploitation [42].

\section{A. SIMob Evolutionary Test-bed}

In the framework of the SIMob project, a prototypal version of the Architectural Framework, named SIMob Evolutionary Test-Bed, has been implemented and deployed by leveraging on the commercial ESB.

The Test-bed has been labeled "evolutionary" since it can evolve over time as its constituent elements are added, reconfigured, or replaced, according to a System of Systems perspective.

Fig. 5 shows the current snapshot of the Evolutionary Testbed, which embodies a set of added-value components developed or extended through specific applied research activities, namely: a Traffic Flow Predictor, a multimodal optimal Route Planner, a rule-based Billing Engine for toll applications and a Fleet Management application.

Geospatial Data sources provided by a project partner as well as Tuscany Region's geo-databases and web map service provided by third-parties (e.g., Google Maps, OpenStreetMap) are also included and accessible via standard interfaces.

The above mentioned components are plugged in the Testbed through purposely developed adapters:

- A Traffic Information Adapter that intermediates the access to traffic information providers (e.g., traffic operational centers). The adapter message-based interface has been designed according to the Datex2 standard specifications [16];

- A Gate Adapter providing a uniform interface to access information on vehicle transit at road gates (e.g., toll gates, access control gates, etc.);

- A Geospatial Data Adapter for accessing geo-spatial information based on the Web Map Tile Service (WMTS) specification [43];

- A Public Transport Adapter for accessing planned and real-time information on public transport routes, according to the Service Interface for Real Time Information (SIRI) specifications [15].

At present, the Test-bed is fed with real and simulated data sources according to information requirements posed by ongoing demo applications (described hereafter).

Traffic and transit events are currently provided by simulators, implemented as Java software modules that publish events in the framework. By varying proper configuration parameters (i.e., average values for traffic and transit events and time intervals), the simulation modules can be configured in order to resemble real-life traffic scenarios (e.g., early in the morning, rush hour, holiday) in an area of interest.

To validate the proposed cooperative approach towards innovation, a set of demonstrators has been collaboratively developed and tested by all project partners, in reference 
application scenarios, by exploiting the Test-bed capabilities.

Traffic Zoning. Traffic zoning concerns the adoption of information and communication technologies in conceiving, promoting and enforcing policies for traffic and multi-modal transport management in urban areas. In this context, Test-bed capabilities have been exploited to develop a tolling service that can be configured according to multi-criteria rating policies. This service leverages on transit gate simulators and on the services provided by the Billing Engine component. A video describing the demo is available for download at [44].

City Logistics. City logistics has been defined as "the process for totally optimizing the logistics and transport activities by private companies in urban areas while considering the traffic environment, the traffic congestion and energy consumption within the framework of a market economy" [45]. Infomobility services can help in providing services and information to facilitate urban freight transport, while minimizing negative impacts on the urban area. In this context, project activities focused on the design and development of graphical interfaces for on-board devices in urban goods transport scenarios. This activity was carried out by exploiting traffic information and routing services provided by the Traffic Flow Predictor and the Route Planner.

Location-based services. This demonstrator concerns the delivery of information of interest to a user in relation to his position and surrounding area. This mashup application provides users with a uniform graphical interface enabling access to optimal route plans, traffic predictions and thematic information (e.g., Points of Interest) made available by different providers. This prototype aimed at validating the Test-bed capability of supporting the integration of traffic information sources, traffic prediction and optimal route planning components, and geo-spatial databases.

\section{B. Interoperation with External Systems}

A few activities have been carried out to make the Test-bed infrastructure interoperate with external systems managed by organizations involved in the ITS and Mobility Information Service domain.

An adapter has been developed to interoperate with the Infomobility Center managed by the Tuscany Region [46]. This center acquires and processes data concerning traffic events and infrastructure monitoring on some extra-urban roads and thus it would represent a reliable source of up-todate information about traffic and mobility in the covered areas of the Tuscany region.

Ongoing activities are also running to interoperate with the UIRNET platform. UIRNET is the Italian national platform operating to support integrated logistics and intermodal transport in Italy [47]. The objective of UIRNET is the development of a distributed platform made by national-level and regional nodes delivering integrated services for logistics and intermodal goods transport. The investigation of how the regional Test-bed could interoperate with the UIRNET System is a long-term activity that we are carrying out with the participation of UIRNET representatives and some industrial partners of the Cooperative Network in the framework of some ongoing national research projects.

\section{Implementing and Testing the OWEN-MIS}

Fig. 6 shows how the OWEN-MIS approach has been put into practice for the cooperative conception and development of an added-value application.

More specifically, the diagram depicts the interactions among the Cooperative Network and the Architectural Framework (i.e. the SIMob Evolutionary Test-bed) in the context of the "Location-based services" demo development. It

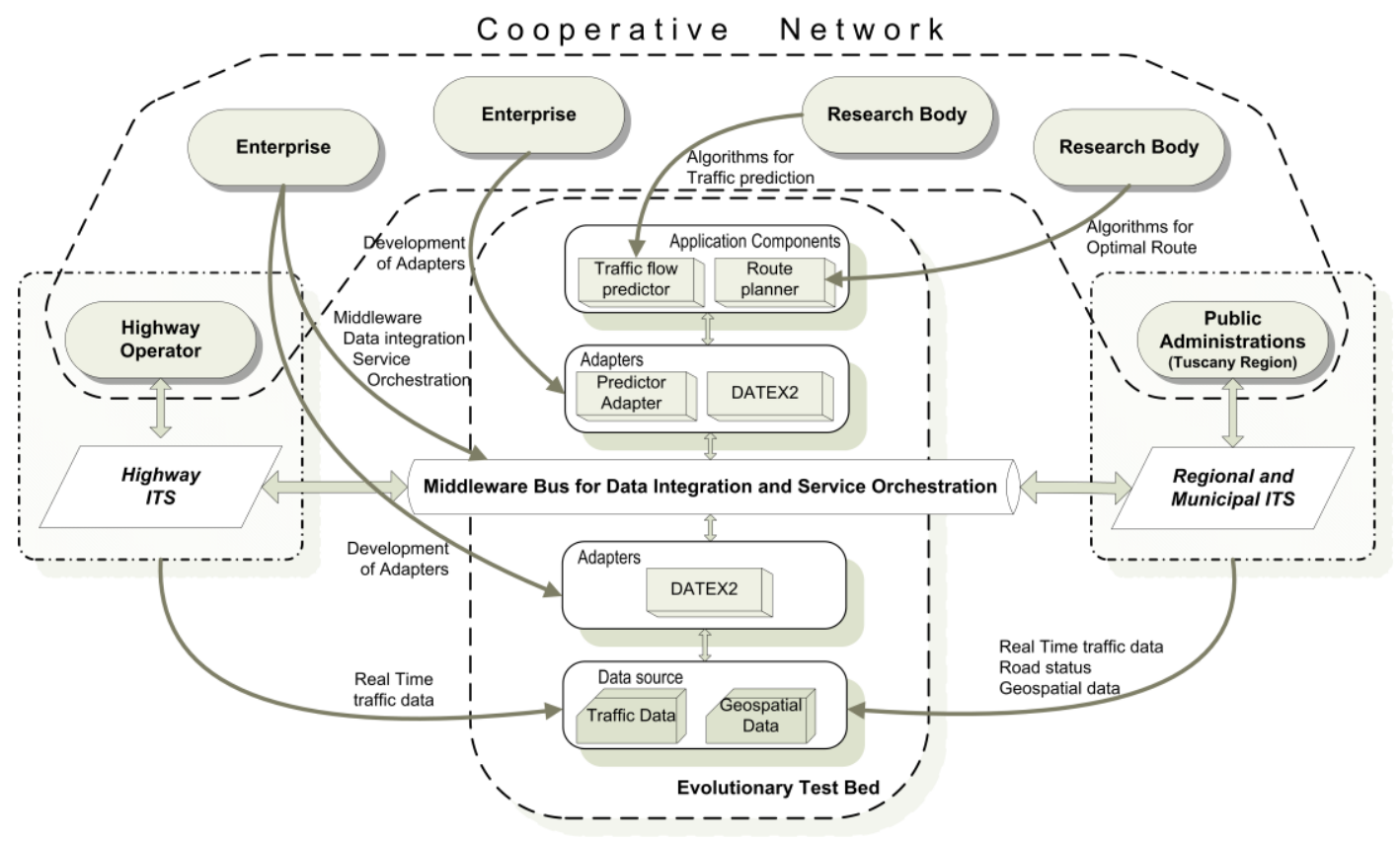

Fig. 6 OWEN-MIS implementation for the "Location-based services" Demo Application 
shows some specific contributions by Cooperative Network members to the demo-application setup. In this context, some components (algorithms and adapters) have been developed purposely for the demo, while some pre-existing systems have been customized for their adoption.

We especially remark the relevance of the role played by two specific Network members, namely a Highway Operator, and the Tuscany Region, which, as owner of ITSs, provided access to their information systems (e.g., geospatial and road databases, traffic and transportation control rooms) to interoperate with the Test-bed on a SoS perspective.

As an overall consideration, we argue that: 1) the addedvalue services provided by the demo application stem out from the synergy of network players and their contributions in terms of incremental innovation, radical innovation and shared information and know-how; 2) such functional and technological evolution of the Framework can attract new members. Indeed, a few enterprises have shown interest in joining the Network. However, long-term sustainability of this approach is matter of study, as discussed in the next paragraph.

\section{DISCUSSION AND CONCLUDING REMARKS}

In this paper we presented the OWEN-MIS, a cooperative approach for promoting and supporting innovation processes in the Mobility Information Service domain.

We first outlined the model of the OWEN-MIS, enabling the creation of synergies in a Cooperative Network of heterogeneous stakeholders: Enterprises, Research Bodies and Public Administrations.

According to roles played by these actors, we elicited main types of outcomes expected as possible results of cooperative networking, thus as factors fostering collaboration.

As part of the Open Working Environment, an Architectural Framework has also been presented. This framework has been conceived as a set of configurable and extensible technological assets for effectively supporting cooperation among heterogeneous actors and speeding up research and development activities. This leads to consider the framework implementation also as an Evolutionary Test-bed that can be properly configured and extended for supporting specific experimental activities, as well agile exploitation of results by involved stakeholders.

The OWEN-MIS aims at enabling interoperability with both existing and new solutions, sub-systems and systems, while accommodating interests and requirements of different players in the mobility scenario. This approach also allows the flexibility needed for possible, sometimes not foreseen, variability of organizational and user requirements as well as technological changes.

A dedicated management structure for the governance of the Network is going to be defined according to its expected evolution along time. In fact, while in the initial transitory phase the Cooperative Network was populated by a restricted group of strongly committed stakeholders, its progressive extension may change the balance of roles and expertise thus requiring an adaptation of the network governance.

Moreover, proper business models are also needed to specify the roles of stakeholders in contributing to and exploiting capabilities provided by the Architectural Framework and easing the specification of plans for shared and single exploitation of results. Experiences carried out within the SIMob project have shown the need for coping with changing requirements and interests of involved enterprises by allowing flexible partnerships and different degrees of innovation openness.

Activities are running to extend the Cooperative Network by involving other interested actors. While activities are ongoing for disseminating results of the SIMob project and make the network grow by attracting new members, work is still needed to guarantee the long-term activity of the Network.

Due to the inherent complexity of the Cooperative Network model, we are aware of the fact that the promotion of the proposed approach shows critical aspects. Particularly, its implementation and long-term sustainability call for proper financial supports and enabling legislation frameworks.

On this direction, the proposed methodological approach is coherent with principles and guidelines of two national funding programs recently promoted by the Italian Ministry for University and Research, calling for cooperative proposals on Smart Cities and Communities [48],[49]. These programs aim at the establishment of national clusters made by large companies, small and medium enterprises, universities and research bodies, and supported by local and national public administrations. Our methodology is also coherent with analogous funding initiatives at European level (e.g. Horizon 2020 Framework Programme). Therefore, we deem that these types of programs can offer financial and legislative actions required for supporting the Cooperative Network implementation at long-term.

The Architectural Framework has been proposed as the technological playground for promoting innovation by achieving different degrees of interoperation across systems, independently managed by different stakeholders. The Architectural Framework has been implemented and deployed as an "Evolutionary Test-bed" to host application demonstrators developed by SIMob project partners. We explicitly label the Test-bed "evolutionary" to stress how its structural and functional characteristics evolve with its autonomous constituents.

As no centralized control can be enforced in the OWEN, there must be sufficient cooperation and consistency of actions to cause desired innovation properties to emerge [32]. In this framework, the value of the Test-bed does not consist only in the provided technological assets, rather in the fact that the Test-bed (the current instantiation of the Architectural Framework) is the place where goals, interests and agreements of stakeholders (Cooperative Network) are translated into technological artifacts (components) and contracts (interfaces).

The Test-bed has been designed and implemented by leveraging on SOA principles and on the adoption of the ESB 
pattern. This choice has been motivated by the consideration that SOA specifications are coherent with the assumption that constituent components operate independently and their internal evolution is independent from the other components (supposed that the interface remains the same).

However, we are aware that SOA specifications lack in providing support to model and manage SOS emergent properties [32], representing desired (positive) outcomes, as well as to side effects that can have unpredictable, negative consequences. Discussion is ongoing in the scientific community in order to investigate how SOA models and technologies should evolve and be complemented to effectively support evolutionary and emergent properties of SoS [11], [50]. To this purpose, research directions which should deserve further attention to enhance the Architectural Framework are: formal analysis and verification techniques, to assure the overall system dependability; autonomic paradigms, to model and implement self-management techniques enabling component to achieve their own objectives while cooperating to global ones; dynamic reconfiguration techniques to make components adapt to changes of objectives or surrounding environment [51].

Moreover, in order to guarantee the rational management of the Architectural Framework capabilities and their proper assemblage into coherent application-driven Test-beds, as well as to facilitate the design of interoperation levels with external SoS, we need to adopt a proper description and analysis methodology. Existing Architectural Frameworks (e.g., US DOD Architecture Framework [52] and the ISO Reference Model for Open Distributed Processing, RM-ODP [53]) are potential candidates, however their adequateness to handle complex systems in practice has been not clearly recognized yet [6]. To this purpose, collaboration is in progress with Selex SI, a company involved worldwide in the design and realization of complex SoS for the Homeland Security [54].

\section{ACKNOWLEDGMENT}

The authors would like to acknowledge basic, specific contribution by all SIMob Consortium partners: CNIT National Inter-University Consortium for Telecommunications (Project Coordinator), University of Siena - Dept. of Information Engineering, Institute of Applied Physics "Nello Carrara" (Italian National Research Council), University of Florence - Dept. of Civil and Environmental Engineering, Autostrade per l'Italia S.p.A, Engineering Sanità Enti Locali S.p.A., Elsag Datamat S.p.A., Geoin s.r.1., NEGENTIS s.r.1., Powersoft s.r.l. The authors thank Eng. Paolo Neri, Selex SI for his useful suggestions and Mr. Luca Capannesi for his technical support.

\section{REFERENCES}

[1] EU Commission, "The potential of Intelligent Transport Systems for reducing road transport related greenhouse gas emissions", Special Study No. 02/2009, December 2009. Available: http://ec.europa.eu/enterprise/archives/e-business-

watch/studies/special_topics/2009/documents/SR02-2009_ITS.pdf
[2] Commission of the European Communities, ““'Keep Europe moving Sustainable mobility for our continent", Comm. from the Commission to the Council and the European Parliament, COM 314 final, 2006.

[3] EU Commission, A sustainable future for transport. Towards an Integrated, Technology-Led and User-Friendly System, 2009. http://www.ec.europa.eu/transport/publications/doc/2009_future_of_tra nsport_en.pdf .

[4] James M. Parker, "Applying a System of Systems Approach for Improved Transportation", S.A.P.I.EN.S, [Online], $3.2 \mid 2010$, Online since 09 septembre 2010. Available: http://sapiens.revues.org/1011.

[5] M. W. Maier, "Architecting principles for systems-of-systems", Systems Engineering, vol. 1, 1998, pp. 267-284.

[6] M. W. Maier, "Research Challenges for Systems-of-Systems" IEEE Int. Conf. on Systems, Man and Cybernetics, 2005, vol.4, pp. 3149- 3154.

[7] M. Ottens, M. Franssen, P. Kroes, I. Van De Poel, "Modelling infrastructures as socio-technical systems", International Journal of Critical Infrastructures, Volume 2, Number 2-3 / 2006

[8] A. Mostafavi, D. M. Abraham, D. DeLaurentis, and J. Sinfield, "Exploring the Dimensions of Systems of Innovation Analysis: A System of Systems Framework", IEEE Systems Journal, vol. 5, no. 2, 2011, pp. 256-265.

[9] J. W.Forrester, Industrial Dynamics, Pegasus Communication, 1961

[10] D. DeLaurentis and R. K. C. A. Callaway, "System-of-systems perspective for public policy decisions," Rev. Policy Res., vol. 21, no. 6, pp. 829-837, 2004.

[11] R. Calinescu, M. Kwiatkowska, "Software Engineering Techniques for the Development of Systems of Systems", in Foundations of Computer Software. Future Trends and Techniques for Development, Lecture Notes in Computer Science, 2010, vol. 6028, pp. 59-82.

[12] A. Tuominen, T. Ahlqvist, "Is the transport system becoming ubiquitous? Socio-technical roadmapping as a tool for integrating the development of transport policies and intelligent transport systems and services in Finland", Technological Forecasting and Social Change, vol. 77, no. 1, 2010, pp.120-134.

[13] C. Freeman, Technology Policy and Economic Performance: Lesson From Japan. London, U.K.: Frances Pinter, 1987

[14] Transport Research Knowledge Centre Official Web Site, Available: http://www.transport-research.info/web/projects/transport_themes.cfm

[15] Service Interface for Real Time Information (SIRI) Web Site [Online]. Available: http://www.kizoom.com/standards/siri/

[16] Datex2 Official web site [Online]. Available:http://www.datex2.eu/

[17] US DOT. The National ITS Architecture http://www.its.dot.gov/arch/

[18] S.S. Saad, "ITS in Japan, a different approach to transportation policy", World Review of Intermodal Transportation Research, vol. 1, no. 1, 2006, pp. 45-54.

[19] X. Wang, "ITS Technology and Policy in China", in the Proc. of the International Conference on ITS Telecommunications, 2006.

[20] European ITS Framework Architecture, Official Web Site. Available: http://www.frame-online.net/

[21] ARchitettura Telematica Italiana per il Sistema dei Trasporti (ARTIST), Official Web Site. Available: http://www.its-artist.rupa.it

[22] Framework Architecture for ITS Design in France, Official Web Site. Available: http://www.its-actif.org/?lang=en

[23] ARKTRANS, Official Web Site. Available: http://arktrans.no/english

[24] Coopers (CO-OPerative SystEms for Intelligent Road Safety) Project, Official Web Site. Available: http://www.coopers-ip.eu/

[25] CVIS (Cooperative Vehicle-Infrastructure Systems) Project, Official Web Site. Available: http://www.cvisproject.org

[26] In-Time Project, Official Web Site. http://www.in-time-project.eu/

[27] Open Knowledge Definition Web Page [Online]. Available: http://opendefinition.org/

[28] European Commission, "Open data -An engine for innovation, growth and transparent governance", Communication from the Commission to the European Parliament, 12.12.2011, COM(2011) 882 final, Available: http://ec.europa.eu/information_society/policy/psi/docs/pdfs/opendata20 12/open_data_communication/en.pdf

[29] E. Almirall, R. Casadesus-Masanell, "Open versus Closed Innovation: A Model of Discovery and Divergence", Academy of Management Review, 2010, Vol. 35, No. 1, 27-47.

[30] European Commission "2003/7: SMEs in Europe 2003". Available: http://ec.europa.eu/enterprise/policies/sme/files/analysis/doc/smes_obser vatory_2003_report7_en.pdf 
[31] M. Lazzeroni, C. Marullo, A. Patrono, R. Perugi, A. Piccaluga, "L’Alta Tecnologia in Toscana, Imprese e territori", $2^{\circ}$ Rapporto annuale, Osservatorio sulle Imprese High-Tech della Toscana, 2011. Available: http://www.osservatorio.sssup.it/documenti/pubblicazioni/Unioncamere 2011.pdf

[32] D. Fisher, "An Emergent Perspective on Interoperation of Systems of Systems, Technical Report no. CMU/SEI-2006_TR-003, Carnegie Mellon University, Pittsburh, USA

[33] M.P. Papazoglou, P. Traverso, S. Dustdar, F. Leymann, "ServiceOriented Computing: State of the Art and Research Challenges," Computer, vol.40, no.11, pp.38-45, Nov. 2007.

[34] M.-T. Schmidt, B. Hutchison, P. Lambros, and R. Phippen. 2005. The enterprise service bus: making service-oriented architecture real. IBM Syst. J. 44, 4 (October 2005), 781-797.

[35] A. Thomas, T. Turner, S. Soderlund, Net-Centric Adapter for Legacy Systems, IEEE Systems Journal, 3, 3, 8 (2009).

[36] G.S. Blair, M. Paolucci, P. Grace, and N. Georgantas, "Interoperability in Complex Distributed Systems", in Proc. SFM, 2011, pp.1-26.

[37] M. Happner, R. Burridge, and R. Sharma. Sun Microsystems. Java Message Service Specification, 2000 http://java.sun.com/products/jms

[38] Object Management Group, Business Process Modeling Notation (BPMN) Specification, Feb. 2006, www.bpmn.org.

[39] F. Curbera, M. Duftler, R. Khalaf, W. Nagy, N. Mukhi, S. Weerawarana, "Unraveling the Web services web: an introduction to SOAP, WSDL, and UDDI" IEEE Internet Computing, vol.6, no.2, pp.86-93, 2002.

[40] D. Parlanti, F. Paganelli, D. Giuli, "A Service-Oriented Approach for Network-Centric Data Integration and its Application to Maritime Surveillance", IEEE Systems Journal, vol. 5, no.2, 2011.

[41] F. Paganelli, D. Parlanti, "A Dynamic Composition and Stubless Invocation Approach for Information-Providing Services", IEEE Transactions on Network and Service Management, 2013 doi: 10.1109/TNSM.2013.022213.120229.

[42] D. Giuli, F. Paganelli, S. Cuomo, P.Cianchi, "A Systemic and Cooperative Approach towards an Integrated Infomobility System at Regional Scale", in the Proc. of 11th Int. Conf. on ITS Telecommunications (ITST 2011), art. no. 6060117, pp. 547-553, St. Petersburg, August 2011.

[43] Open GeoSpatial Consortium, OpenGIS Web Map Tile Service Implementation Standard. Available: http://www.opengeospatial.org/standards/wmts

[44] SIMob Project, Traffic Zoning Demo. Available: http://www.negentis.com/download/simob/SIMOB.wmv

[45] E. Taniguchi, R. G. Thompson, T. Yamada, Modelling city logistics, City logistics, pp. 3-37. Kyoto: Inst. of Systems Science Research, 1999

[46] Regione Toscana, Infomobilità, Official Web Site. Available: http://www.regione.toscana.it/trasporti/infomobilita/index.html

[47] UIRNET Official Web Site, http://www.uirnet.it

[48] Italian Ministry for University and Research, "Smart Cities and Communities and Social Innovation" Call for Proposals [Online]. Available: http://attiministeriali.miur.it/anno-2012/luglio/dd05072012.aspx

[49] Italian Ministry for University and Research, "National Technological Clusters" Call for Proposals [Online]. Available: http://attiministeriali.miur.it/anno-2012/maggio/dd-30052012.aspx

[50] S. Selberg, M.A. Austinm, "Toward an Evolutionary System of Systems Architecture", Eighteenth Annual Int. Symp. of The Int.Council on Systems Engineering (INCOSE), Utrecht, The Netherlands, 2008.

[51] I. Zappia, F. Paganelli, D. Parlanti "A lightweight and extensible Complex Event Processing system for sense and respond applications", Expert Systems with Applications, vol. 39, no. 12, 2012, pp. 1040810419

[52] Department of Defense Architecture Framework Working Group, DoD Architecture Framework Version 1.0, USA, 2004.

[53] ISO/ITU-T, Reference Model for Open Distributed Processing (ISO/ITU-T 10746 Part 1 - 4), 1997.

[54] D. Giuli, F. Paganelli, P. Neri, "Integrated Infomobility Systems: Experimenting an Open Technical and Cooperative Approach to Support Continuous Innovation and Evolution", Polaris Innovation Journal, SELEX Sistemi Integrati, Tech. Review, no. 8, pp.56-62, 2011. 